\title{
Über einige alte und neue Ascidien aus dem Zoologischen Museum von Amsterdam.
}

\author{
VON \\ G. PH. SLUITER, \\ o. Professor an Der Universttät Amsterdam.
}

(Mit Tafel 1).

Die auf den folgenden Seiten erwähnten, teilweise als neu beschriebenen Ascidien fanden sich noch in den Vorräten des Amsterdammer Zoologischen Museums, das in den Gebäuden der Königlichen Zoologischen Gesellschaft "Natura Artis Magistra" untergebracht ist. Auch meinerseits ergreife ich freudigst diese Gelegenheit diese Zeilen meinem. Freunde, dem hochgesinnten Director dieser Gesellschaft, zur Feier seines '70sten Geburtstages zu widmen. Dass die so überaus glückliche Verbindung des Zoologischen Instituts der Universität mit der Königlichen Zoologischen Gesellschaft in den vielen Jahren so reiche Früchten getragen hat, ist wohl in erster Linie dem weiten Blick Kerberts zu danken. Die lebende Habe der Gesellschaft hat selbstverständlich in erster Linie seine Fürsorge, aber auch Museum und Laboratorium erfreuen sich immer seiner warmen Teilname. Möge es ihm vergönnt sein noch manche Jahre im Interesse der Zoologie und unserer Amsterdammer Institute wirksam zu sein.

Von den unten aufgeführten 15 Arten, habe ich 6 als für die Wissenschaft neue Formen beschrieben und eine als neue Varietät. Es sind dies: Polycarpa vankampeni, Distomus malayensis, Polyandrocarpa robusta, Phallusia julinea, Polycitor glabrun, Polycitor giganteus und die neue Varietät: Distomus hupferi Mchlsn., var. compacta. Besonders möchte ich auf die beiden Formen Phallusia julinea und Polycitor giganteus aufmerksam machen, welche einige merkwürdige anatomische Verhältnisse aufweisen.

Pyura Hupferi Mchlsn.

Michaelsen. Die Pyuriden des Naturhist. Museums zu Hamburg. Mitt. aus dem Naturh. Museum. XXV. Hamburg 1908, p. 238 und „Beiträge zur Kenntniss der Meeresfauna West-Afrikas. Tunicaten." Hamburg 1915, p. 371.

Mehrere Exemplare dieser sehr typischen und leicht kenntlichen Art wurden von Herrn Vermeulen an der. Senegal-Küste gesammelt. Der ausführlichen Beschreibung Michaelsen's ist kaum etwas hinzuzufügen, nur sei erwähnt, dass bei meinen Exemplaren die Fühler nur sehr schwach verästelt sind, so dass bei den meisten die Verästelungen 2ter Ordnung schon fehlten. Ferner erscheinen auch die Falten am Kiemensack etwas weniger breit, da ich nur 14-18 Längsgefässe zählen konnte, während bei den Exemplaren von Michaelsen 20-28 vorkamen. Sonst stimmen meine Exemplaren genau mit der Beschreibung Michaelsens überein. 
Pyura spinosa Sluiter.

Sluiter. Tuniciers recueillis en 1904, par M. Ch. Gravier, dans la Golfe de Tadjourah (Somalie française). Mém. Soc. Zoologique de France. 1905. T. 18, p. 16.

Die 3 mir jetzt von der Maurischen Küste vorliegenden Exemplare sind beträchtlich grösser als das früher von mir beschriebene Tier, da das grösste Exemplar $42 \mathrm{~mm}$ lang, $30 \mathrm{~mm}$ breit und $20 \mathrm{~mm}$ dick ist. Die Siphonen sind bis $8 \mathrm{~mm}$ lang und zwar ist der Branchialsipho immer breiter als der Atrialsipho. Alle Tiere sind wieder mit der dorsalen rechten Seite auf Schwämmen festgewachsen. Die linke, so wie auch die ventrale, rechte Seite ist ganz nackt, ohne Fremdkörper. Auch sind bei diesen grösseren Tieren mit weit offen stehenden Öffnungen, diese letzeren viel ausgeprägter vierlappig. Die eigentümlichen, stachelförmigen Schuppen, welche die ganze Oberfläche bedecken, haben zwar in Hauptsache dieselbe Gestalt wie früher von mir beschrieben wurde, aber sie scheinen bei den mehr ausgewachsenen Exemplaren ihre Form doch etwas zu ändern. So sind bei den kleinen Schuppen, welche den ganzen Körper bedecken, die $\mathbf{5}$ bis 7 Dornen an der Basis weniger nach innen gebogen, auch sind sie mehr gestreckt. Die grösseren Stacheln, welche mehr vereinzelt, wenn auch in ziemlich grosser Anzahl über den Körper verteilt sind, sind auch etwas anders gestaltet als bei dem früher beschriebenen kleineren Tiere. Sie sind nämlich beträchtlich stärker und mehr breit konisch, während sie bei dem Djibouti-Exemplar schlank waren. Dasselbe gilt auch für die grösseren Stacheln, welche auf dem Siphonen und namentlich am Rande der beiden Öffnungen besonders auf den 4 Lappen vorkommen. Endlich sei noch erwähnt, dass auch an der Innenseite der beiden Siphonen 4 Längsreihen von Schuppen vorkommen, welche die gleiche Gestalt besitzen wie die Schuppen an der Aussenseite, nur sind sie mehr bräunlich gefärbt und tragen eine etwas grössere Zahl secundärer Dörnchen an der Basis.

Für die Flimmergrube sei noch bemerkt; dass bei einem Exemplar das rechte Horn nach aussen, das linke nach innen eingerollt ist. Der Kiemensack hat im allgemeinen den Bau wie ich ihn für das kleinere Tier von Djibouti beschrieben habe, nur hat die Zahl der Kiemenspalten in den Maschen etwas zugenommen, so dass die mehr dorsal gelegenen Maschen von 10 oder 11 Spalten durchbrochen sind, die mehr ventral liegenden nur von 6 oder $\%$ Zwischen 2 Falten kommen gewöhnlich 4 Längsgefässe vor. Von den 16 Fühlern möchte ich noch hervorheben, das sich nur Verästelungen 2ter Ordnung finden und diese letztere sogar nur kleine stummelförmige Fortsätze darstellen.

Polycarpa asymmetrica Sluiter.

Styela asymmetrica Sluiter. Die Tunicaten der Siboga-Expedition I, pg. 8\%.

Es liegen mir 3 Exemplare dieser Art vor, welche Prof. Dr. P. N. van Kampen in 190\%, während seiner Fahrten mit dem "Gier" in der Java-See sammelte, und zwar auf $6^{\circ} 26^{\prime} \mathrm{S}$. und $112^{\circ} 41^{\prime}$ O., aus einer Tiefe von 30 Faden. Die Tiere stimmen im Allgemeinen sehr gut mit den Siboga-Exemplaren von der Insel Gebé überein, nur möchte ich ein paar Ergänzungen hinzufügen. Der Zellulosemantel ist bei diesen 3 Exemplaren dünner als bei den Siboga-Exemplaren und auch weniger fest. Für den Kiemensack gilt als vollständiges Schema: E. $6|y| 6|6| 5 \mathrm{D}: 3|6| 6|y| 6 \mathrm{E}$, wobei also besonders auffällt, dass rechts von der Dorsalfalte 5 und links 3 Längsgefässe auftreten. Anf die Falten kommen 8 oder 9 Längsgefässe. Die Anordnung der Quergefässe entspricht dem Schema: 143424341, wobei die Gefässe 4 ter Ordnung zuweilen noch als parastigmatische Gefässchen erscheinen, während ich bei den Siboga-Exemplaren keine solche fand. Für den sehr geräumigen Darm sei noch erwähnt, dass der Afterrand nicht ganz glatt ist, sondern sehr wenig tief wellenförmig eingeschnitten. Die Gonaden sind, wie ich es früher angab, fast ganz in das Gewebe der inneren musculösen Mantels versenkt, so dass sie kaum in den Peribranchialraum hervorragen. Endocarpen scheinen auf den ersten Blick ganz zu fehlen, aber schon bei Lupenvergrösserung sieht man zahlreiche, ganz kleine Bläschen von etwa $0.2 \mathrm{~mm}$ hervorragen, welche bei microscopischer 
Untersuchung sich als wirkliche, ganz kleine Endocarpen kund geben. Die asymmetrische Lage der Flimmergrube kommt auch hier vor, wenn auch nicht so auffällig wie bei den Siboga-Exemplaren.

Polycarpa aurata $Q$ \& $\mathrm{G}$.

Für die Literatur siele: Sluiter. Tunicaten der Siboga-Expedition I, fig. 5\%.

Mehrere Exemplare dieser Art wurden von Herrn van der Sande auf dem Küstenriffe der Insel „Pulu Wèrè" an der Nord-Küste von Flores gesammelt. Sie gehört wohl zu dem meist verbreiteten Polycarpa-Arten im Indischen-Archipel und ist so wohl durch den äusseren Habitus als auch durch ihren anatomischen Bau leicht kenntlich, wenn auch der letztere vielfach kleine Abweichungen zeigt. Den früheren Beschreibungen ist nur wenig hinzuzufügen; nur für den Kiemensack sei noch bemerkt, dass die breiten Falten 20 bis 22 Längsgefässe besitzen und dass gewöhnlich die Maschen, welche den Falten am nächsten liegen breiter sind als die, welche in der Mitte des Feldes zwischen zwei Falten liegen, so dass erstere von 6 oder 7 Spalten durchbrochen sind, während die mittleren Maschen nur 3 oder 4 besitzen.

Für die Flimmergrube sei noch bemerkt, dass sie bei allen diesen Exemplaren von Flores im eine grosse Anzahl von gesonderten Öffnungen aufgelöst ist. Der eigentümliche Bau des Zellulosemantels mit den zahlreichen kolbenförmigen Anschwellungen der Blutgefässe tritt auch bei all diesen Exemplaren deutlich hervor. Nur die Farbe des Zellulosemantels ist fast völlig verschwunden, so dass die Tiere jetzt ganz blass gelblich erscheinen.

Polycarpa aurita Sluiter.

Sluiter. Natuurk. Tijdschr. v. Nederl. Indië Bd. 50, p. 338.

Sluiter. Die Tunicaten der Siboga-Expedition I, p. 59.

Ein Exemplar dieser früher von mir beschriebenen Form wurde von van Kampen auf $6^{\circ} 8^{\prime} \mathrm{S}$. und $108^{\circ} 18^{\prime}$ O., aus einer Tiefe von 16 bis 18 Faden gesammelt. Es gehört das Tier jedenfalls zu den weniger seltenen Formen im Malayischen Archipel.

Polycarpa vankampeni n. sp. Fig. 1, 2.

Äussere Kennzeichen. Das grösste der beiden vorliegenden Tiere ist $35 \mathrm{~mm}$ lang, $20 \mathrm{~mm}$ breit und $10 \mathrm{~mm}$ dick. Vorn ist der Körper schief abgestumpft, so dass die Branchialöffnung auf der vorderen Spitze liegt und die Atrialöffnung auf $2 / 5$ der Körperlänge weiter nach hinten, von welcher Stelle an der Körper gleich breit bleibt. Beide Öffnungen sind deutlich 4-lappig. Die Tiere sind mit der hinteren rechten Seite auf Sand, Korallen-Bruchstücken u. s. w. festgewachsen, sonst ist die Oberfläche ganz glatt und auch nur sehr oberflächlich und spärlich gefurcht. Die in Alkohol aufbewahrten Tiere sind jetzt milchich weiss gefärbt.

Der Zellulosemantel ist fest, lederartig und ziemlich dick.

Der Innenkörper besitzt eine kräftige Muskulatur, und zwar ist besonders die äussere Ringmuskelschicht gut entwickelt. Die beiden Siphonen sind kurz und breit und wenig deutlich vom übrigen Körper abgesetzt.

Der Kiemensack besitzt die gewöhnlichen 4 Falten, welche ziemlich schmal sind und 10 bis 12 Längsgefässe tragen. Die Zahl der Längsgefässe zwischen den Falten entspricht dem Schema: D. 3|5-7|4|5|5-6. E. Die Quergefässe sind von verschiedener Breite, nach dem Schema 13231 angeordnet, aber die Gefïsse $2^{\text {ter }}$ und $3^{\text {ter }}$ Ordnung unterscheiden sich nur wenig in Grösse, während diejenigen der 1sten Ordnung sehr viel breiter sind. In den Maschen kommen regelmässig 8 bis 10 Kiemenspalten vor, Der Endostyl ist schmal und wenig hervortretend.

Die Flimmergrube ist hufeisenförmig, das rechte Horn aber biegt mit einer längeren sich schlängelnden Schleife entweder nach innen oder nach aussen. (Fig. 1, 2).

Die Dorsalfalle ist sehr schmal und glattrandig.

Der Darm bildet die gewöhnliche Doppelschlinge, ist überall sehr geräumig so dass der 
Magen nur wenig scharf angedeütet ist. Der Enddarm ist nur kurz, was mit der ziemlich weit nach hinten liegenden Atrialöffnung zusammenhängt und der Afterrand ist tlef eingeschnitten, so dass er mit einem Kranz von fingerförmigen Papillen umstellt erscheint.

Die Fühler sind ungefähr 50 in Anzahl, wovon etwa 40 grössere. Letztere sind ziemlich lang und schmal.

Die Gonaden sind als ziemlich zahlreiche, unregelmässig angeordnete, birn- oder eiförmige Polycarpen entwickelt. Sie sind ungefähr $2 \mathrm{~mm}$ lang und sind nur sehr lose mit dem inneren Mantel verbunden, so dass sie sehr leicht abreissen. Das zentral liegende grosse Ovarium wird von den. Testesbläschen und den verästelten. Vasa efferentia umgeben. Die Öffnungen der kurzen Oviducte und Vasa deferentia liegen im Allgemeinen dem Atrialsipho zugewendet, wenn auch die Längsachsen der Polycarpen einander nicht immer ganz parallel verlaufen.

Fundort. Javameer, $5^{\circ} 44^{\prime}$ S. und $114^{\circ} 22^{\prime}$ O., Tiefe 32 Faden (Ex. A.); $0^{\circ} 14^{\prime} \mathrm{N}$., und $104^{\circ} 4^{\prime}$ O., Tiefe 13-16 Faden (Ex. B.). Beide Exemplare gesammelt von van Kampen, während den Fahrten des Dampfer's "Gier."

Die beiden Exemplare stimmen in Hauptsache gut mit einander überein, nur ist bei dem einen (Ex. A) das Horn der Flimmergrube nach aussen, bei dem andern (Ex. B) nach innen gekehrt. Ferner finden sich bei Ex. A. 7 Längsgefässe zwischen der 1 sten und $2^{\text {ten }}$ Falte und bei Ex. B. nur. 5 .

Obgleich keine besonders auffällige Eigentümlichkeiten in ihrem Bau zeigend, kann ich diese beide Tiere doch nicht zu einer der zahlreichen Polycarpa-Arten bringen, die jetzt schon aus dem Malayischen Archipel bekannt sind.

Die ziemlich grosse Zahl der Fühler, die Anordnung der Längsgefässe in dem Kiemensack, der fingerförmig eingeschnittene Afterrand u. s. w. fand sich in dieser Kombination bei keiner der früher von mir beschriebenen Formen.

Distomus (Alloéocarpa) Hupferi Mchlsn., var. compacta n. var. (Fig. 3-6).

Äussere Kennzeichen. Die Koloniën bilden flach ausgebreitete, oder auch gebogene Platten von 2-4 $\mathrm{mm}$ Dicke, welche auf Bryozoen und Muschelschalen aufgewachsen sind. An der Oberfläche ragen die Einzeltiere, dicht gedrängt neben einander liegend, etwas hervor, besonders aber der Rand um die beiden Öffnungen. Dieser aufstehende Rand ist jetzt tief radiär gefurcht. Bei dem stark contrahierten Zustande ist in diesen Furchen nur schwierig zuweilen ein vierlappiger Einschnitt zurück zu finden. Die Öffnungen selbst sind kreisrund. Grössere und kleinere Tiere liegen unregelmässig verteilt neben einander. Die Grössten sind an der Oberfläche $3 \mathrm{~mm}$ lang, während dann die beiden Öffnungen $2 \mathrm{~mm}$ von einander entfernt sind. Die Farbe der in Alkohol aufbewahrten Exemplaren ist grau mit deutlich violettem Schimmer.

Die Einzeltiere werden bis $3 \frac{1}{2} \mathrm{~mm}$ lang und besitzen den typischen Habitus der Polyzoinen. Die Muskulatur ist ziemlich kräftig, so dass der Kiemensack nur undeutlich durchschimmert. Die Siphonen sind sehr kurz.

Der Zellulosemantel ist sehr fest lederartig, aber zwischen den Einzeltieren nur als dünne Lamellen entwickelt, an der Basis der Kolonie eine dickere zusammenhängende Platte bildend.

Der Kiemensack besitzt jederseits im Ganzen 11 Längsgefässe, welche in recht ungleichen Abständen. von einander entfernt sind und 'zwar so, dass von der Dorsalfalte abgerechnet zuerst 8 Kiemenspalten auftreten ohne Unterbrechung durch ein Längsgefäss, dann folgen 5 Längsgefässe dicht neben einander mit nur je einer Kiemenspalte zwischen sich, die man also als .1ste Falte ansehen kann. Darauf folgen wieder 5 Kiemenspalten, dann ein Längsgetäss, dann 6 Kiemenspalten. Dann folgt eine 2te rudimentäre Falte aus 3 Längsgetässen bestehend, die 1 resp. 2 Kiemenspalten zwischen sich frei lassen. Dann folgt eine Reihe von 9 nicht unterbrochenen Kiemenspalten, dạn ein Längsgefäss, 4 Kiemenspalten, 1 Längsgefäss, 3 oder 4 Kiemenspalten und zuletzt der Endostyl. Die Quergetässen sind ungetähr gleich breit; sehr regelmässig überbrücken parastigmatische Quergefässchen die Kiemenspalten. (Fig. 3). 
Die Dorsalfailte bildet ein schmaler, glattrandiger Saum:

Die Flimmergrube ist ein schwach gebogener quer verlaufender Schlitz.

Die Fühler sind 16 in Anzahl, und zwar wechseln regelmässig 8 grosse mit 8 kleinen ab.

Der Darm macht, wie gewöhnlich eine enge Schleife an der. linken Seite des Kiemensackes. Der Magen besitzt regelmässig 12 tiefe Falten, von welchen, wie auf dem Querschnitt. leicht ersichtlich, jederseits 5 gleich tiefe kommen, dann eine: sehr breite am hinteren Medianrand und eine etwas schmälere am Vorderrande. Die Falten sind nicht gerade, sondern mit schwacher spiraliger Biegung. Die Afterrand ohne Einschnitte. (Fig. 4).

- Die Gonıden wie typisch für Alloeacarpa, rechts die Ovarien, links die Hodenbläschen und. zwar so, dass die Ovarien sich mehr nach hinten, die Hodenbläschen sich mehr im Vorderteil anhäufen. Bei jungen Tieren sind die kleinen Ovarien in einfacher Reihe neben den Endostyl angeordnet, die Hodenbläschen als kleine ovoide Säckchen in abwechselnd gegen. einander gedrungener Doppelreihe. Bei erwachsenen Tieren bleiben die Ovarien fast kugelrund; die Hodenbläschen aber werden zu längeren, schlauchförmigen Organen, mit verhältnissmässig langem Vas deferens mit etwas trichterförmig erweiterter Öffnung. (Fig. 5, 6).

Fundort. Cap Blanco, W. Afrika 1. Kol.; Senegal 1 Kol.; Bai du Levrier, W. Afrika 1 Kol. Alle gesammelt von Herrn Vermeulen aus einer Tiefe von \pm 15 . Faden.

Der anatomische Bau der Einzeltiere dieser Kolonien gleicht fast ganz dem von. Alloeocarpa Hupferi Mchlsn. und es ist nur der ganz verschiedene äussere Habitus, der mich veranlasst diese neue Varietät auf zu stellen. Während doch nach Michaelsen's Diagnose bei A. hupferi die Kolonie besteht: ", aus vollständig von einander gesonderten, in ganzer Länge frei aufragenden, eiförmigen bis kugeligen, im Maximum $3 \frac{1}{2} \mathrm{~mm}$ langen und $21 / 2 \mathrm{~mm}$ dicken Personen," so sind bei unserer : neuen Varietät die. Tiere fast ganz. in dem gemeinschaftlichen Zellulosemantel eingebettet und ragen nur sehr wenig an der. Oberfläche hervor. Der Zellulosemantel bildet denn auch eine dicke, feste Masse an der Unterseite nnd keine „dünnen, plattenförmigen oder schmälere bandförmigen Stolonen", wie bei den Kolonien von Michaelsen: Dies so ganz verschiedenes Äusseres lässt denn auch sehr wenig vermuten, dass wir mit so gleichförmig gebauten Tieren zu thun haben. Nur der. Bau des Kiemensackes und die Zahl der Fühler zeigen einige Unterschiede. Eine zweite rudimentäre Falte scheint bei A. Hupferi zu fehlen und auch die Zahl der Kiemenspalten zwischen den Längsgefässen ist bei den beiden Formen verschieden. Dann erwähnt Michaelsen 32 Fühler, während ich nur 16 fand. Allerdings treten hier und dort ganz winzige Fühlerchen als Andeutungen von Fühlern $3^{\text {ter }}$ Ordnung auf, was die Zahl ebenfalls auf 32 bringen würde, wenn diese alle sich weiter entwickelten. Sonst stimmen beide Formen in Betreff des. Baues des Magens so wie des Geschlechtsapparat völlig mit einander überein.

Da auch die von Michaelsen beschriebene Grundform von West-Afrika, Gorée bei Cap Verde, stammt, so ist es wohl am wahrscheinlichsten, dass unsere Form nur eine compacte Varietät desselben vorstellt, was vielleicht mit dem etwas verschiedenen Bau des Kiemensackes zusammenhängt.

Distomus (Aloeocarpa) malayensis n. sp. (Fig. \%, 8).

Äussere Kennzeichen. Die Kolonie bildet eine flach ausgebreitete Kruste, welche stellenweise bis $6 \mathrm{~mm}$ dick wird und auf Bryozoënstöcken festgewachsen ist. An der freien Oberfläche ragen die Einzeltiere ziemlich weit kuppenförmig hervor, so dass sie in dem wahrscheinlich. ziemlich stark contrahierten Zustande noch bis zu $3 \mathrm{~mm}$ hoch hervorstehen. Die beiden Öffnungen liegen etwa $1 \mathrm{~mm}$ von einander entfernt auf der abgestumpften Spitze dieser kuppenförmigen Hügel; sie sind beide kreisrund, von einem vielfach strahlich gefurchten Rande umgeben, ohne dass aber. grössere Einschnitte zur Bildung von 4 Lappen zu unterscheiden sind. Die Oberfläche dieser Hügel ist nicht glatt, sondern mit kleinen Höckerchen bedeckt, die allerdings erst bei Lupenvergrösserung deutlich sichtbar werden. Die Farbe der in Alkohol aufbewahrten Kolonie ist grau mit schwach violettem Schimmer. 
Der Zellulosemantel ist fest und zähe. Er besitzt die gewöhnliche Structur, so dass nur sternförmige Testazellen aber keine Blasenzellen vorkommen.

Die Einzeltiere sind derartig in den Zellulosemantel orientiert, dass der Dorsalteil mit den beiden Siphonen in den hervorragenden Hügelchen liegen, der Ventralteil mit Endostyl und dem Darm in dem compacten Teil des Mantels. Die Länge der grösseren Individuen ist $3 \mathrm{~mm}$, die dorso-ventrale Breite ungefähr $4 \mathrm{~mm}$.

Der Innenkörper ist mit kräftiger Muskulatur versehen.

Der Kiemensack ist ohne eigentliche Falten, da jederseits, statt deren, nur 3 Längsgefässe vorkommen, welche in ungleicher Entfernung von einander liegen, so dass vom Endostyl nach der Dorsalfalte zu die Felder zwischen denselben immer schmäler werden. So kommen zwischen Endostyl und erstem Längsgefäss 9 Kiemenspalten vor, zwischen diesem und dem $2^{\text {ten }}$ Längsgefäss 5 , im dritten Felde 4 oder 3 und endlich zwischen dem am meisten dorsalen Längsgefäss und der Dorsalfalte nur noch 2 Kiemenspalten. Die Kiemenspalten selbst sind lang und schmal und fast immer von parastigmatischen Quergefässchen überbrückt. Die übrigen Quergetässe sind ungefähr gleich breit. (Fig. \%).

Die Flimmergrube ist wie gewöhnlich bei den kleinen Formen, kreisrund, höchstens etwas breiter als lang.

Die Dorsalfalte ist schmal und glattrandig.

Die Fïhler sind 16 in Anzahl, wovon 8 grössere mit 8 kleinen abwechseln.

Der Darm liegt, wie typisch für die ganze Familie, linksseitig neben dem hinteren Teil des Kiemensackes. Der kurze Oesophagus mündet in den länglich-runden Magen. Letzterer besitzt 14 ziemlich tiefe Falten. Der übrige Darm macht die gewöhnliche Schlinge und ist geräumig. Der Enddarm ist kurz und mündet mit einem weit offen stehenden After, dessen Rand nur schwach wellenförmig eingeschnitten ist.

Die Gonaden sind jederseits des Endostyls, links und rechts als kleine Polycarpen entwickelt. Rechts liegen einige wenige Ovarien, von welchen ich meistens zwei oder sogar nur eins fand, zuweilen aber auch drei. Links fand ich ziemlich regelmässig 6 Hodenbläschen in einer Reihe hinter einander neben dem Endostyl, von welchen das vorderste und das hinterste beträchtlich kleiner sind, etwa halb so gross, wie die 4 mittleren. Die Öffnungen der Oviducte sind nach vorn, die der Vasa deferentia dem Endostyl zugekehrt. (Fig. 8).

Fundort: Java-See, $6^{\circ} 26^{\prime}$ S., $112^{\circ}-41^{\prime}$ O., Tiefe 30 Faden, gesammelt von van Kampen, während den Fahrten des Dampfers „Gier” am 14 Dez. $190 \%$.

Es ist dies eine ganz typische Distomus-Art, bei der die Kiemenfalten ganz zurück: gebildet sind und nur noch 3 einfache Längsgetässe erhalten geblieben sind. Ferner ist noch hervorzuheben, dass die Zahl der Ovarien stark reduziert ist und sogar bis auf eins herabsinken kann, während sonst bei Distomus gewöhnlich diese Zahlenreduction den Hodenbläschen zufällt.

Polyandrocarpa robusta n. sp. (Fig. 9-12).

Äussere Kennzeichen. Die einzige vorliegende Kolonie bildet eine dünne polsterartige Masse von $5^{1 / 2} \mathrm{~cm}$ Länge, und $4 \frac{1}{2} \mathrm{~cm}$ Breite, während die Dicke an verschiedenen Stellen zwischen $6 \mathrm{~mm}$ und $10 \mathrm{~mm}$ schwankt. Mit der breiten Unterseite war die Kolonie auf Sand, KorallenBruchstücken u.s.w. festgewachsen. An der freien Oberfläche erscheinen die Einzeltiere als etwas dunklere Flecken von 4-6 $\mathrm{mm}$ Länge und 3-4 $\mathrm{mm}$ Breite, welche scharf von den zwischen gelegenen Partieën des Zellulosemantels abgegrenzt sind, welche heller, weisslichgrau gefärbt sind und dadurch ein heller gefärbtes Netzwerk zwischen den Einzeltieren bilden. Eine Anordnung der Tiere zu besonderen Systemen kommt nicht vor. Die beiden Öffnungen sind ungefähr $2 \frac{1}{2} \mathrm{~mm}$ von einander entfernt. Beide sind vierlappig, was aber bei der ziemlich starken Kontraktion der Tiere nicht immer gleich deutlich zu Tage tritt. Die Oberfläche ist nicht ganz glatt, sondern mit kleinen Wärzchen bedeckt, welche nur in der Umgebung 
der beiden Öffnungen etwas grösser werden, sonst nur mit der Lupe zu unterscheiden sind. Der Zellulosemantel ist fest und zähe, knorpelig und grau weisslich gefärbt ohne Pigment. Es kommen in dem Gewebe nur die sternförmigen Testazellen vor, keine Blasenzellen.

Die. Einzeltiere haben eine ovoide Gestalt mit einer Längsachse bis zu $5 \mathrm{~mm}$ und einer Breiteachse von 3 bis $4 \mathrm{~mm}$. Die Siphonen sind kurz und der ganze Innenkörper mit mässig kräftiger Muskulatur versehen.

Der Kiemensack besitzt jederseits 4 Falten, von welchen aber die $2^{\text {te }}$ und $4^{\text {te }}$ von der Dorsalfalte abgerechnet, viel schwächer entwickelt sind als die beiden anderen. Die Verteilung der Längsgefässe entspricht folgendem Schema: D 1 (10) 2 (4-6) 1 (10-12) 3 (4-6) - E. Zwischen der Dorsalfalte und erstem Längsgefäss liegen 8 Kiemenspalten, zwischen Endostyl und der $4^{\text {ten }}$ Falte 5 Kiemenspalten. In den übrigen Feldern wechselt die Zahl zwischen 2 und 4, wie es aus der Abbildung zu ersehen ist. Die Kiemenspalten selbst sind gewöhnlich ziemlich breit, etwa 4 mal so lang als breit, wenigstens in den breiteren Feldern. Die Quergefässe sind ungefähr gleich breit, parastigmatische Quergefässchen kommen vielfach vor. Der Endostyl ist mässig breit. (Fig. 9).

Die Flimmergrube bildet eine lange schmal-ovale Spalte. (Fig. 10).

Die Dorsalfalte ist glattrandig und verhältnissmässig breit.

Die Fühler sind lang und schmal. Es kommen im Ganzen ungefähr 40 vor, welche nur wenig verschieden sind in Länge, obgleich eine Abwechselung von etwas grösseren und kleineren öfters noch zu unterscheiden ist.

Der Darm liegt wie gewöhnlich linksseitig gegen den hinteren Teil des Kiemensackes. Der ungefähr birnförmige Magen besitzt im Ganzen 14 tief ins Innere hineinragende Falten. Am hinteren Teil des Magens liegt der hakenförmig gebogene Blindsack. Der geräumige Mittel- und Enddarm biegt sich hinter dem Magen in eine enge Doppelschlinge, deren Schenkel unmittelbar gegen einander liegen. Der After ist glattrandig. (Fig. 11).

Die Gonaden sind jederseits als mehrere, länglich runde, hermaphroditische Polycarpen entwickelt, welche jederseits des Endostyls eine Reihe von $y$ oder 8 Drüsen bilden. Die grössten sind $0.87 \mathrm{~mm}$ lang und halb so breit. Die ungefähr 18 Hodenbläschen, welche unter dem Ovarium liegen sind ungefähr so gross wie die grössten Eier im Ovarium. (Fig. 12).

Fundort. Java-See $4^{\circ} 25^{\prime}$ S., $116^{\circ} 2^{\prime}$ O., gesammelt von van Kampen während den Fahrten des Dampfers „Gier" am 12 Dez. 1908, Tiefe: 15-20 Faden.

Obgleich der Bau der Einzeltiere nur wenig abweicht von dem der beiden Arten, welche die Siboga-Expedition erbentete, so kann ich doch die eben beschriebene Form nicht mit diesen identifizieren. Erstens ist der Habitus der ganze Kolonie mit dem kräftig entwickelten Zellulosemantel recht verschieden, dann bleiben auch die Einzeltiere beträchtlich kleiner und ist auch der Bau des Kiemensacks mit den reduzierten $2^{\text {ten }}$ und $4^{\text {ten }}$ Kiemenfalten zu abweichend um als blosse Variante zu gelten. Die Polyandrocarpa lapidosa von Herdman, welche Michaelsen nachuntersuchte hat nur 11 Falten am Magen und einen eingeschnittenen Afterrand und weicht überhaupt, auch durch den mit Sand inkrustierten Zellulosemantel mehr von den Indischen Formen ab.

Phallusia julinea n. sp. (Fig. 13-16).

Äussere Kennzeichen. Das einzige erbeutete Exemplar ist $10 \mathrm{~cm}$ lang und über die grösste Länge ungetähr $3 \mathrm{~cm}$ breit, nnr hinten etwas breiter, seitlich etwas zusammengedrückt, aber immerhin noch $2 \mathrm{~cm}$ dick. Nach vorn zu biegen die beiden kräftigen Siphonen nach der Dorsalseite über; der Branchialsipho ist ungefähr $3 \frac{1}{2} \mathrm{~cm}$, der Atrialsipho ungefähr $21 / 2 \mathrm{~cm}$ lang. Die beiden Öffnungen sind deutlich 10-lappig eingeschnitten, während die 10 Furchen noch für ungefähr $1.5 \mathrm{~cm}$ auf der Oberfläche zu verfolgen sind. Diejenigen des Branchialsiphos sind dann scharf vom übrigen Körper abgegrenzt, die des Atrialsiphos verstreichen mehr allmählig. Das Tier ist mit der hinteren linken Seite auf Korallen-Bruchstücken und Muschelschalen festgewachsen. Die sonstige Oberfläche ist ganz glatt, obne Fremdkörper, nur 
mit wenig ausgesprochenen Furchen, welche wohl durch Kontraktion verursacht sind. Die Farbe ist halbdurchscheinend grau, wie typisch für die meisten Phallusia und Ascidia-Arten. (Fig. 13).

Der Zellulosemantel ist mässig dick, halb-knorpelig, mit der typischen Struktur des AscidiaMantels, mit zahlreichen grossen Blasenzellen und spindelförmigen Testazellen.

Der Innenkörper, aus dem Zellulosemantel herausgenommen, misst $8.5 \mathrm{~cm}$, wovon $4 \mathrm{~cm}$ auf den schmalen Vorderteil kommen, welcher wie ein langer Sipho aussieht, und $4 \frac{1}{2} \mathrm{~cm}$ auf den breiteren Hinterkörper. Der Atrialsipho misst jetzt $2.8 \mathrm{~cm}$. Die Muskulatur der rechten Seite ist mässig kräftig entwickelt, die der linken Seite nur kräftig an den beiden langen Siphonen. Die Eingeweide schimmern im Hinterkörper deutlich durch. Das Ganglion liegt weit nach hinten etwas vor der Umbiegungstelle von Atrialsipho in Branchialsipho. (Fig. 14).

Der Kiemensack ist sehr regelmässig gebaut; er zeigt eine recht deutlich ausgeprägte Längsfältelung, wodurch immer die Kiemenspalten unterhalb der schmalen Längsgefässe, sehr schmal erscheinen und schwierig zu zählen sind. Sonst liegen regelmässig zwischen zwei Längsgefässe 3 breite Kiemenspalten, welche ungefähr 6 mal so lang als breit sind. Von den schmal erscheinenden Spalten, welche in der Längsfältelung liegen scheinen jeweilen ungefähr 6 .vorzukommen. Die Quergefässe unterscheiden sich nur sehr wenig in Breite, wenn auch hier und dort die gewöhnliche Abwechselung von breiteren und schmäleren etwas deutlicher zu Tage tritt. Auf den Kreuzpunkten stehen ziemlich kräftige konische Papillen, welche aber an der Basis noch regelmässig einige, etwa 3 oder 4, kleinere. Nebenzäckchen besitzen. Intermediäre Papillen habe ich nirgends gefunden. Parastigmatische Quergefässchen kommen hier und dort vor, aber nur ganz vereinzelt. (Fig. 15).

Die Flimmergrube ist völlig verschwunden, ja die ganze Stelle, -wo die Flimmergrube zu erwarten wäre und wo sonst die Peripharyngeallippen nach hinten ausbiegen, ist ganz schmal geworden, da die Dorsalfalte weit nach vorn vordringt und die Peripharyngeallippen fast den Fühlersaum berühren. Statt der Flimmergrube sind aber mehrere sekundäre, dorsale Öffnungen eutwickelt, welche in den Peribranchialraum ausmünden. (Fig. 16).

Die Dorsalfalte ist ziemlich breit, über die ganze Länge glattiandig aber deutlich gerippt.

Die Fühler sind 40 in Anzahl, zwar ungleich lang, aber ohne dass ein bestimmtes Regelmass in der Anordnung zu entdecken ist.

Der Darm macht die gewöhnliche Doppelschlinge. Die erste Schlinge reicht ziemlich weit nach vorn, bleibt nur wenig hinter dem Winkel zwischen den beiden Siphonen zurück. Bei der hinteren Umbiegung der zweiten Schlinge bildet der Darm wieder eine sackartige Erweiterung, wodurch der Enddarm nur mässig lang ist. Der glattrandige After liegt an der Basis des Atrialsiphos.

- Die Gonaden liegen wie gewöhnlich hauptsächlich in der ersten Darmschlinge, nur die Verästelungen der Hoden greifen auf der rechten Seite der Darmwand über. Ein sehr dickes, weiss gefärbtes Vas deferens und ein schmäleres, weniger helles Oviduct folgt den Lauf der zweiten Darmschlinge und beịde münden neben dem After in den Atrialraum.

Fundort. Insel Nassi besar in der Java See, Tiefe 9 Faden, gesammelt durch van Kampen während den Fahrten des Dampfers "Gier".

Es ist dies jedenfalls eine typische Phallusia-Art in Sinne Julin's, wie auch Hartmeyer diese Abgrenzung der beiden Gattungen Ascidia und Phallusia übernommen hat und wie ich meine ganz mit Recht. Auffallend ist die Zehnzahl der Lappen um die beiden. Öffnungen, welche Zahl auch bei der früher von mir beschriebenen Ascidia decemplex (Natuurk. Tijdsch. $\dot{v}$. Nederl. Indië, Bd. L, p. 343) vorkommt. Der ganze Habitus aber, mit den langen Siphonen und besonders der sehr verschiedene Bau des Kiemensackes, trennt die beiden Arten doch wieder weit von einander. Am merkwürdigsten war mir aber das Verhalten am Vorderende der Dorsalfalte. Die Praebranchialzone wird hier ganz' schmal und die Dorsalfalte dringt fast bis zum Fühlersaum vor, während die Peripharyngeallippen stark nach vorn 
biegen, anstatt, wie es gewöhnlich der Fall ist, nach hinten, zur Bildung des dreieckigen Feldes, in welchem das Dorsaltuberkel mit der Flimmergrube sonst liegt. Die bekannte Erscheinung bei einigen Ascidiidae, wobei die Flimmergrube bei den erwachsenen Tieren allmählig kleiner wird und sich sekundäre in den Peribranchialraum ausmündende Kanälchen und Öffnungen bilden, ist also hier zum Extrem durchgeführt, da die Flimmergrube völlig verschwunden ist. Die Zahl der sekundären Kanälchen und Öffnungen in den Peribranchialraum habe ich an dem einzigen Exemplar nicht feststellen können. Ich schliesse mich der Ansicht Julin's, welcher auch Hartmeyer geneigt ist an zu erkennen, an, um die Ascidiidae, wobei diese eigentümliche Umbildung der Abfuhrcanäle der Neuraldrüse vorkommt, in die Gattung Phallusia zu vereinigen und möchte ich diese Art Julin widmen, der so viel zur Klarstellung dieser Frage beigetragen hat.

Polycitor scaber Sluiter.

Sluiter. Die Tunicaten der Siboga-Expedition II, fig. 25.

Eine Kolonie dieser Art sammelte van Kampen, während seiner Fahrten mit dem Dampfer "Gier" auf dem Riffe der Insel Pulu Paree, eine der "Tausend Inseln" in der Java-See. Die Kolonie stimmt genau mit meiner früheren Beschreibung des Tieres von der Insel Jedan überein.

Polycitor segmentatus Sluiter.

Sluiter. Die Tunicaten der Siboga-Expedition II, fig. 9.

Mehrere Kolonien sammelte van Kampen, ebenfalls auf dem Riffe Pulu Paree, zusammen mit der vorigen Art. Sowohl das Äussere der Kolonie als auch der Bau der Einzeltiere stimmen mit meiner früheren Beschreibung überein. Allein die Tiere sind noch etwas länger und damit ist der stielförmige Abschnitt des Abdomens, zwischen dem Thorax und dem angeschwollenen Hinterteil des Abdomens mit dem Magen und Gonaden dünner als bei den Siboga-Exemplaren. Ich vermute, dass auch das Fehlen der eigentümlichen Einschnürungen, welche ich damals beschrieb, bei den jetzt vorliegenden Exemplaren dadurch verursacht wird, dass die Tiere weniger contrahiert sind.

Da ich die Kolonien mit den Originalen der Siboga-Expedition nochmals verglichen habe, und sonst keine nennenswerten Unterschiede sehe, sind die Einschnürungsringe bei den Siboga-Exemplaren wohl mehr als zufällige Erscheinungen auf zu fassen.

Polycitor (Eudistoma Caull.) glabrum n. sp. (Fig. 17).

Äussere Kennzeichen. Die grösste der 3 vorliegenden Kolonien ist $6{ }^{1} / 2 \mathrm{~cm}$ lang, $4 \mathrm{~cm}$ breit und $3 \mathrm{~cm}$ hoch. Sie bilden dicke, polsterartige Massen, welche mit der mehr abgeflachten Unterseite auf den'schlammigen Boden mittelst verschiedener Fremdkörper fest gewachsen waren. Die ganze Kolonie ist gallertig, halbdurchscheinend, die Oberfläche erscheint unter der Lupe feinkörnig, während die Umgebung der Branchialöffnungen mit den 6 papillenartigen Lappen etwas mehr hervorragen. Die Branchialöffuungen sind ungetähr $3 \mathrm{~mm}$ von einander entfernt. Die Atrialöffnungen sind ebenfalls 6 lappig aber viel undeutlicher und überhaupt öfters viel schwieriger zn entdecken. Die ganze Oberfläche erscheint in unregelmässige Vielecken geteilt, welche mit der Lage der Einzeltiere übereinkommen und welche Zeichnung verursacht wird durch die ziemlich dünnen Lamellen des Zellulosemantels zwischen den Einzeltieren. Bei schräg auffallendem Lichte ist diese eckige Zeichung besonders deutlich zu sehen.

Die Einzeltiere sind alle sehr stark zurückgezogen, so dass die Lücken in dem oberen Teil des Mantels, wo einmal die Thoraces lagen, jetzt alle leer sind. Jetzt sind die Einzeltiere nnr noch $8 \mathrm{~mm}$ lang aber waren im Leben jedenfalls doppelt so gross, wenn man den Abstand misst von der Branchialmündung an der Oberfläche bis an die Stelle wo jetzt die Abdomina liegen. Jetzt kommen auf den Thorax $3 \mathrm{~mm}$, auf den Stiel und das Abdomen $5 \mathrm{~mm}$. Die Muskulatur ist sowohl am Thorax als am Abdomen ziemlich kräftig. Die beiden 
kurzen Siphonen stehen beide am Vorderrande des Tieres, der Atrialsipho nur etwas weiter nach hinten. Beide Öffnungen erscheinen deutlich 6-lappig. (Fig. 17).

Der Zellulosemantel ist gallertig und ziemlich weich, bildet an der oberflächlichen Schicht nur dünnere Platten zwischen den Thoraces der Einzeltiere und eine mehr compacte Masse in den tieferen Schichten, wo die Stiele und Abdomina der Personen liegen. Besonders in der mehr oberflächlichen Schicht kommen, ausser den gewöhnlichen spindelförmigen Testazellen, dicht gedrängt grosse Blasenzellen vor, welche ein Durchmesser von $0.1 \mathrm{~mm}$ erreichen. Auch in den tieferen Schichten finden sich noch Blasenzellen, aber viel weniger gehäuft. Hier sind aber zahlreichen Kotballen angehäuft, die vielleicht von abgestorbenen Tieren herstammen.

Der Kiemensack besitzt 3 Reihen von lang ausgezogenen Kiemenspalten, die bei den stark contrahierten Tieren stark und unregelmässig eingeschrumpft erscheinen.

Die Dorsalfalte besteht nur aus 2 langen, dreieckigen Zungen.

Die Flimmergrube bildet eine länglich-ruide Öffnung.

Der Darm fängt an mit einem langen weiten Ösophagus, welcher in den fast kugeligen, glattwandigen Magen übergeht. Bald hinter dem Magen erweitert sich der Mitteldarm zur Bildung des Nebenmagens, welcher als ein scharfkantiger; ringförmiger Wulst sich vortut, wie es öfters der Fall ist, vielleicht aber nur durch Contraction diese Form annimmt. Hinter dem Nebenmagen setzt sich der Mitteldarm noch eine kurze Strecke nach hinten fort um dann nach vorn in den Enddarm umzubiegen, welcher ganz mit Kotballen gefüllt ist. Der in zwei Zipfel geschlitzte Afterrand liegt halbwegs der 2ten Kiemenspaltenreihe.

Die Fiihler sind 16 in Anzahl, wovon 8 grössere mit 8 kleineren abwechseln.

Die Gonaden wie gewöhnlich in der Darmschlinge.

Fundort: Java-See, $6^{\circ} 37^{\prime}$ S., $109^{\circ} 16^{\prime}$ O. Tiefe 20 Faden, gesammelt von van Kampen, während den Fahrten des Dampfers „Gier”. 3 Ex.

Anfangs war ich geneigt diese drei Kolonien. zu meiner früher beschriebenen Art $P$. mollis (Die Tunicaten der Siboga-Expedition II, p. 11) zu stellen, aber nachdem ich die beiden Originalen nochmals mit einander verglichen hatte, scheint mir das doch nicht statthaft. Erstens ist der ganze Habitus nicht gleich, da bei $P$. mollis von einer Felderung an der Oberfläche nichts zu sehen ist, während andrerseits bei unserer neuen Art, die Einzeltiere gar nicht durchschimmern. Das wäre nun aber von untergeordneter Bedeutung, aber auch in dem inneren Bau treten Unterschiede auf. Die Kiemenspalten sind bei $P$. mollis viel kürzer, in dem Zellulosemantel fehlen Blasenzellen und Kotballen und ein Nebenmagen scheint dort nicht entwickelt. Es sind dies wohl genügende Gründe beide Arten anzuerkennen.

Polycitor (Paradistoma Caull.) giganteus n. sp. (Fig. 18-20).

Äussere Kennzeichen. Die einzige mir vorliegende Kolonie bildet eine feste, dick cylindrische Masse von $12 \mathrm{~cm}$ Höhe und ungefähr $8 \mathrm{~cm}$ in Durchmesser. Die Kolonie war offenbar mit der Basis auf Steinchen, Muschel-Bruchstücken u. s. w. festgewachsen, welche noch an der Basis befestigt sind. Die Oberfläche des in Alkohol aufbewahrten Exemplars ist jetzt bräunlich, im allgemeinen glatt, nur an der oberen freien Fläche hängen zahlreiche, mehr oder weniger regelmässig gestaltete, freie, zungenförmige Fetzen des Zellulosemantels. Es ist mir nicht möglich zu entscheiden, ob dies dem ursprünglichen Zustand entspricht, oder ob sich diese Fetzen erst nachträglich bei der Conservierung los getrennt haben. Die Einzeltiere sind nicht in Systeme geordnet und gemeinschaftliche Cloackalöffnungen fehlen vollständig. Die beiden Öffnungen der Einzeltiere sind kreisrund und deutlich an der Oberfläche zu erkennem.

Der Zellulosemantel ist ziemlich fest, mehr oder weniger knorpelig, mit schwach angedeuteter faseriger Structur in der Richtung der Längsachsen der Einzeltiere. Blasenzellen konnte ich nicht mit Sicherkeit unterscheiden. Das bräunliche Pigment dringt nur sehr wenig weit in das Innere durch, so dass nur die äusserste Oberfläche braun erscheint, die ganze innere Masse aber hell weisslich grau gefärbt ist. Nur am Rande der oberen und unteren Fläche dringt die braune Farbe etwas weiter vor. 
Die Einzeltiere erreichen jetzt noch die enorme Grösse von $4 \frac{1}{2} \mathrm{~cm}$ und sind deutlich in Thorax, Halsteil und eigentliches Abdomen geteilt und zwar so, dass auf jedem dieser 3 Körperabschnitte regelmässig $1 \frac{1}{2} \mathrm{~cm}$ kommt. Jedenfalls waren aber die Tiere stark zusammengezogen so dass sie im Leben noch beträchtlich länger waren. Die beiden ungefähr $2 \frac{\mathrm{mm}}{2}$ langen Siphonen sind nach vorn gekehrt und münden beide direct an die Oberfläehe aus. Die Öffnungen sind nur sehr undeutlich 6-lappig, erscheinen fast kreisrund, nur dass 6 Bündel von etwas kräftigeren Längsmuskeln eine Andentung der Sechszahl geben. Die Muskulatur des Innenkörpers ist besonders am Thorax kräftig entwickelt, so dass der Bau des Kiemensackes nicht durchschimmert; am Abdomen ist sie viel schwächer, sodass der Verlauf des Darmes leicht zu verfolgen ist. (Fig. 18).

Der Kiemensack ist gross und besitzt jedenfalls nicht weiniger als 40 Reihen von Kiemenspalten. Die Kiemenspalten sind lang, schmal und gerade gestreckt: An den Quergefässen kommen kräftige, zungenförmige Fortsätze vor, in Zahl mit den Kiemenspalten übereinkommend. genau wie es sich bei der Gattung Glossophorum verhält. Der Endostyl ist von normaler Breite und durch die starke Kontraction des Thorax mit gedrungen-schlängelndem Verlauf. (Fig. 19).

Die Flimmergrube ist verhältnissmässig gross und zeigt die typische $U$-Form vieler einfachen Ascidien. Die Öffnung des U-förmigen Bogens ist nach rechts gekehrt, indem das ganze Gebilde schief zur Längsachse steht. Die beiden Hörner sind schwach nach aussen gebogen. (Fig. 20).

Die Dorsalfalte besteht aus zahlreichen lang-zungenförmigen Fortsätzen.

Die Fühler sind 32 in Anzahl und sehr regelmässig angeordnet, so dass ich immer 8 sehr grosse Fühler fand, dazwischen 8 von 2 ter Ordnung und endlich mit diesen beiden abwechselnd noch 16 beträchtlich kleinere 3 ter Ordnting.

Der Darm fängt an mit einem langen und engen Ösophagus um im Vorderteil des angeschwollenen Abdomens in den länglich runden, nicht grossen Magen aus zu münden. Die Wand des Magens ist in Falten gelegt und zwar mögen etwa 8 vorkommen, welche aber schwer zu zählen sind, da sie nicht gerade gestreckt verlaufen, sondern in ziemlich gedrungenen Schlängelungen liegen, was aber vielleicht durch Kontraktion verursacht ist. Hinter dem Magen setzt sich der Darm bis ganz hinten im Abdomen fort, biegt dann nach vorn, verschmälert sich alsbald, während der enge Enddarm wieder durch den Stiel nach vorn zieht um in den glattrandigen After weit vorn im Thorax aus zu münden.

Die Gonaden liegen im Abdomen in der Darmschlinge und zwar füllt das voluminöse Ovarium fast den ganzen Raum, welcher von der Darmschlinge frei gelassen wird. Der Hoden ist etwas heller gefärbt und umgreift teilweise das Ovarium. Das Vas deferens ist weiss und besonders der Anfangsteil ist deutlich geschlängelt, während es im Stiel mehr gerade verläuft.

Fundort: West-Indien, ohne genauere Ortsangabe, gesammelt van de Wege, 1 Kolonie.

Es ist dies in mehreren Hinsichten eine sehr merkwürdige Art. Erstens fällt die enorme Grösse der Einzeltiere auf, da die Tiere in dem offenbar stark kontrahierten Zustande noch fast einen balben Decimeter lang werden, und mit diesem Riesenwuchs gehen nun wohl andere Besonderbeiten in dem anatomischen Bau Hand in Hand. So ist die Zahl der Reihen von Kiemenspalten bis auf 40 gestiegen, während die höchste bis jetzt bekannte Zahl bei dieser Gattung 24 war. Auch ist vielleicht die Form der Flimmergrube von der Grösse unserer Tiere abhängig. Bei fast allen in Kolonien lebenden Ascidien bildet die Flimmergrube eine ungefähr kreisrunde oder ovale oder höchstens etwas nierenförmig eingebogene Öffnung, was wohl mit der Kleinheit der Einzeltiere zusammenhängt, wobei dieser primitive Zustand wahrscheinlich erst secundär wiedererlangt ist. Bei unserem so viel grösseren Polycitor giganteus hat die Flimmergrube die typische hufeisenförmige Gestalt angenommen oder vielleicht beibehalten, wie sie so vielfach als Grundform bei den grösseren einfach bleibenden Ascidien auftritt. 
Andrerseits ist das Vorkommen der. zungenförmigen Fortsätze an den Quergetässen des Kiemensacks wohl unabhängig von der Grösse der 'Tiere. Es würde hierbei also ein analoger Fall vorliegen wie bei Polyclinum und Glossophorum, welche letztere Gattung von Lahille bloss auf das Vorkommen dieser Fortsätze hin von Polyclinum abgetrennt wurde. Nun ist aber nach den Untersuchungen Hartmeyer's ') das Verhalten dieser beiden Gattungen ein anderes, als wir bisher meinten, da der Typus der Gattung Polyclinum (Polyclinum saturnium Sav.) auch die Fortsätze an den Quergefässen besitzt und also jedenfalls die Gattung "Glossophorum" Lahille einzuzieben ist. Hartmeyer hat diese Fortsätze dann auch bei mehreren anderen, als Polyclinum beschriebenen, Arten, gefunden und formuliert eine schärfere Diagnose dieser Gattung, wobei auch das Vorkommen dieser Fortsätze darin aufgenommen wird. $\mathrm{Ob}$ hiermit das Richtige getroffen ist, will ich vorläufig dahingestellt sein lassen, nur möchte ich bemerken, dass die meisten von mir beschriebenen Polyclinum-Arten durch den glattwandigen Magen, so wie durch die gedrehte Schlinge des Darmes sich der neuen Fassung der Gattung Polyclinum genau unterordnen, allein fehlen die Fortsätze an den Quergefässen. Nun tut sich hier bei unserem Polycitor giganteus ein analoger Fall vor, da die Tiere ihrem ganzen anatomischen Bau nach zu Polycitor zu rechnen sind, nur dass die Quergefässe mit den zungenförmigen Papillen besetzt sind. Anfangs war ich geneigt desshalb die Form in einem neuen Subgenus, z. B. "Parapolycitor" unter zu bringen, aber. weil eben auch bei Polyclinum dasselbe vorkommt, scheint es mir unstatthaft zu viel Gewicht auf das Vorkommen dieser Papillen zu liegen. Vielleicht ist auch Hartmeyer geneigt nach diesem Befund bei Polycitor seine Diagnose für Polyclinum daraufhin zu modifizieren.

Sycozoa (Colella) umbellata Michlsn.

Michaelsen. Tunicaten der Hamburger Magalhaensischen Sammelreise. Hamburg $190 \%$

Von dieser Art besitzt das Museum 10 grössere und mehrere kleine Kolonien, welche von dem der Wissenschaft leider zu früh entfallenen Dr. H. Kruimel in Punta Arenas gesammelt wurden. Alle Exemplare sind auf einer Algen-Art (Nitophyllum) fest gewachsen. Die Stiele, womit sie an die Algen befestigt sind, werden bis $6 \mathrm{em}$ lang, die Köpfchen bis $2 \mathrm{~cm}$ lang und $1.5 \mathrm{~cm}$ breit. Niemals fand ich verästelte Stiele, wie bei den Exemplaren von Michaelsen; immer trug ein Stiel nur ein einziges Köpfchen. Der Stiel ist am dicksten, nämlich bis $4 \mathrm{~mm}$, etwas unterhalb des Köpfchens um sich dann nach dem Köpfchen zu etwas zu verjüngen, viel beträchtlicher aber nach der Basis zu, wo er auf Nitophyllum befestigt ist, und wo die grössten Stiele nur noch $1 \mathrm{~mm}$ im Diameter besitzen. Die Stiele sind ziemlich hart und sehr zähe. Nach der Vermutung Michaelsens, würden die Stöcke also einjăhrige Kolonien sein, welche in der Winterzeit zu Grunde gehen, während der Stiel überwintert um in der neuen Vegetationsperiode am oberen Ende neue Sprossen zu treiben. Michaelsen hat die kleinen, sprossenden Formen als „forma nov. Kophameli" aufgeführt. Die Gestalt der Köpfchen und die Grösse der Einzelpersonen ist etwas verschieden. Nun finde ich diese Grösse-Verhältnisse ganz so bei meinen kleinen Kolonien, wo auch die Einzeltiere nur 1/3 mm lang sind und ich sehe denn auch keinen Grund die "forma Kophameli" nicht als einfache junge sprossende Kolonien der "forma typica" anzusehen. Der Bau der Personen kommt genau mit Michaelsen's Beschreibung überein, so auch die Anordnung des eigentümlichen weissen Pigments, welches dem ganzen Köpfchen die charakteristische Zeichnung verleiht. Michaelsen fand alle Kolonien treibend an der Meeresoberfläche, und vermutet, dass sie vom Felsen losgerissen waren. Alle von Kruimel gesammelten Exemplaren sind auf Nitophyllum festgewachsen, wodurch es sich besser erklären lässt, dass sie auch mitunter ganz frei schwimmend gefunden werden, nachdem die Alge abgerissen oder verwest ist.

1) Hartmeyer, R. Über einige Ascidien aus dem Golf von Suez. Sitzungsber. der Ges. naturf. Freụnde. Berlin 1915 , No.9. 


\section{TAFELERKLÄ̈UNG.}

TAFEL I.

Fig. 1. und 2. Polycarpa vankampeni n. sp. Zwei verschiedene Formen der Flimmergrube.

Fig. 3. Distomus hupferi Mchlsn. var. compacta n. var. Stück des Kiemensackes.

Fig. 4. Distomus hupferi Mchlsn. var. compacta n. var. Querschnitt durch den Magen.

Fig. 5. Distomus hupferi Mchlsn. var. compacta n. var. Hodenbläschen mit Vas deferens.

Fig. 6. Distomus hupferi Mchlsn. var. compacta n. var. Endostyl mit den daneben liegenden Geschlechtssäckchen.

Fig. \%. Distomus malayensis n. sp. Stück des Kiemensackes.

Fig. 8. Distomus malayensis n. sp. Endostyl mit den daneben liegenden Geschlechtssäckchen.

Fig. 9. Polyandrocarpa robusta n. sp. Stück des Kiemensackes.

Fig. 10. Polyandrocarpa robusta n. sp. Flimmergrube.

Fig. 11. Polyandrocarpa robusta n. sp. Magen und Darm.

Fig. 12. Polyandrocarpa robusta n. sp. Ein Geschlechtssäckchen.

Fig. 13. Phallusia julinea n. sp. Habitusbild in natürlicher Grösse.

Fig. 14. Phallusia julinea n. sp. Der Innenkörper.

Fig. 15. Phallusia julinea n. sp. Stück des Kiemensackes.

Fig. 16. Phallusia julinea n. sp. Peripharyngealsaum mit Fühlerkrani und vorderer Spitze der Dorsalfalte, ohne Flimmergrube.

Fig. 1\%. Polycitor glabrum n. sp. Ein Einzeltier.

Fig. 18. Polycitor giganteus n. sp. Ein Einzeltier.

Fig. 19. Polycitor giganteus n. sp. Stück des Kiemensackes.

Fig. 20. Polycitor giganteus n. sp. Flimmergrube. 


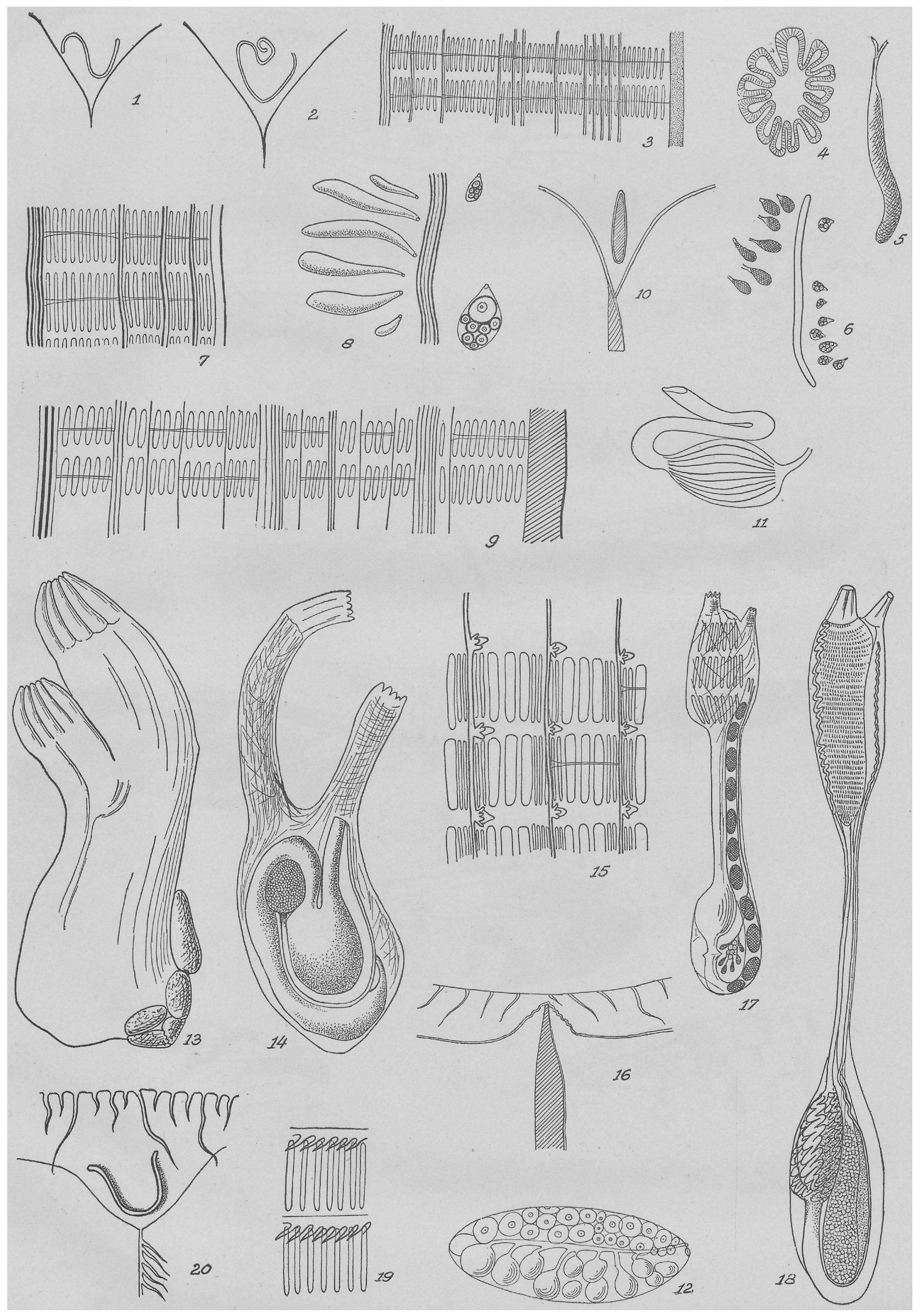

